\title{
Does Michigan Have the Longest Survival Time? A Comparative Analysis of Breast Cancer Patients
}

\author{
Hafiz MR Khan ${ }^{1 *}$, Kemesha Gabbidon², Anshul Saxena², LisaAnn S Gittner ${ }^{3}$, Aamrin Rafiq ${ }^{4}$, Taylor \\ Lenzmeier $^{5}$, Rachel Smith ${ }^{1}$, Sarah Mende ${ }^{1}$, Kandi Quesada ${ }^{1}$, Summre Blakely ${ }^{1}$, Mahlet Afesse ${ }^{1}$, Tiffany \\ Torres $^{1}$, and Maria Lyuksyutova ${ }^{6}$ \\ ${ }^{1}$ Department of Public Health, Texas Tech University Health Sciences Center, Texas, USA \\ ${ }^{2}$ Department of Health Promotion and Disease Prevention, Florida International University, FL, USA \\ ${ }^{3}$ Department of Political Science, Texas Tech University, Texas, USA \\ ${ }^{4}$ Department of Computer Science, Texas Tech University, Texas, USA \\ ${ }^{5}$ Garrison Institute on Aging, Texas Tech University Health Sciences Center, Texas, USA \\ ${ }^{6}$ School of Medicine, Texas Tech University Health Sciences Center, Texas, USA.
}

Received: August 08, 2016; Accepted: September 25, 2016; Published: October 25, 2016

*Corresponding author: Hafiz MR Khan, Department of Public Health, Texas Tech University Health Sciences Center, Lubbock, Texas 79430, USA. E-mail: hafiz.khan@ttuhsc.edu

\begin{abstract}
Background: Breast cancer diagnoses have increased by $40 \%$ across the globe, making breast cancer a serious women's health care and public health concern. The United States, as well as many other developed nations, experiences high breast cancer incidence and mortality rates. This study examines the differing characteristics of breast cancer patients and their survival times for four randomly selected states in the United States: Hawaii, Michigan, Utah, and Washington.
\end{abstract}

Materials and Methods: A Simple Random Sampling (SRS) method was used to select the records of 2,000 breast cancer patients from the aforementioned four states. The SRS method was also used to extract the demographic and disease characteristics from within each of the four states using the Surveillance Epidemiology and End Results (SEER) database. The Cox Proportional Regression was used to compare the breast cancer patient survival times of the selected states.

Results: The probability sample for each of the four states included 2,000 breast cancer cases from the SEER database (1973-2012). Of the four states, Washington had the highest mean age of diagnosis at 61.46 years (Standard Error $[\mathrm{SE}]=0.321$ ), whereas Hawaii presented with the lowest mean age at 58.64 years $(\mathrm{SE}=0.310)$. Mean survival days (in months) were also calculated and stratified by each cancer registry. Michigan reported the longest mean survival time of 152.67 months ( $\mathrm{SE}=14.955$ ), and Hawaii reported the shortest mean survival time of 118.21 (SE=4.861) months, representing a 34.46 months' difference (2.87 years).

Conclusions: Results from this study demonstrate that geographic location and ethnicity affect patient survival and diagnosis. The findings also emphasized the importance of early detection and treatment in reducing the incidence of breast cancer nationwide. Furthermore, the outcomes and methodologies used in this study can be used to guide healthcare providers and researchers in breast cancer treatment, intervention, and prevention.

Keywords:Breast Cancer Data; Incidence Rate; Mortality; Race and Ethnicity; Breast Cancer Survival;

\section{Introduction}

Breast cancer is the most common cancer in women worldwide and it is the second leading cause of cancer death in the United States. Between the years 1975 and 1995, dramatic increases in breast cancer incidence was seen in the United States and other developed nations [1]. During the same time many countries experienced a $20 \%$ to $40 \%$ increase in breast cancer cases, primarily among women over age 50 [1]. In 2008, nearly 1.4 million women were diagnosed with breast cancer and at least 459,000 succumbed to the disease worldwide [24]. As of 2011, breast cancer accounted for $23 \%$ of all cancer cases and $17 \%$ of all cancer related deaths in the world [8]. Nations in the Western Hemisphere face a four-fold higher incidence of breast cancer compared to eastern nations [1]. This difference stems from developing nations facing a lower five-year survival rate whereas developed nations face a higher incidence of the disease [24]. For example, some of the poorer nations in Africa have only a $12 \%$ five-year survival rate, while wealthier nations such as Australia and Canada have approximately a $90 \%$ five-year survival rate [24]. Explanations for this difference in five-year survival rates between developed and developing nations is due to differences in breast cancer screening and access to appropriate health care $[22,24]$. Access to screening and health care are often related to the socioeconomic standing of patients as well as the overall economic standing of the nation. Countries with more than $20 \%$ of their population at or below the poverty level have a $13 \%$ higher death rate from breast cancer and a 3\% higher overall death rate in women compared to developed nations [22]. The most important method for reducing breast cancer mortality is early screening; many nations and organizations have focused on improving screening efforts in order to reduce the incidence of breast cancer mortality. 
In the United States, rates of mammography screening have increased dramatically among women over the age of 40 [5]. Many developing nations have moved toward improving screening efforts, but lack the funds necessary to increase screening programs. To fill in the gaps, many organizations have provided assistance to resource poor nations, such as the Breast Cancer Global Initiative and the National Breast and Cervical Cancer Early Detection Program (NBCCEDP) [3]. In 2002, Susan G. Komen for the Cure and the Fred Hutchinson Cancer Research Center created the Breast Health Global Initiative. The organization has raised \$6 million to improve education and outreach in low resource settings [3]. The NBCCEDP has made efforts to increase breast and cervical cancer screening rates among financially disadvantaged women. The program successfully screened approximately half a million women between the ages of 40 and 64 during 2011 and 2012; unfortunately, this only covers $10 \%$ of the eligible population [7]. States in this program were able to screen an average of $13.7 \%$ of eligible women per state ranging from $3.2 \%$ to $52.8 \%$. A study utilizing SEER and Medicare-linked database findings showed that $43.5 \%$ of the 137,274 eligible women in the cohort had at least one mammogram screening. Additionally, women 66 to 74 years old were more likely to get a mammography screening compared to those 85 to 100 years or older (57.2\% vs. $15.2 \%$, respectively; $p<0.001$ ) [6].

Despite improvements in screening and treatment, there are still racial and ethnic differences in breast cancer screening and mortality in the United States. Mammography use was lowest among American Indians and Alaskan Natives at 36\% [7, 22]. Breast cancer screening rates have remained relatively low in African Americans and Hispanics resulting in greater public health support for increased cancer screening for these groups [13]. Racial and ethnicity-based disparities are not the only factors affecting breast cancer disease and treatment; social, environmental, and hereditary determinants also play a role in the development of breast cancer $[1,5,15,20,22]$.

African-American, American Indian/Alaskan Native, and Asian and Pacific-Islander women have lower five-year survival rates than non-Hispanic White women. African-Americans have the highest death rates of any of the groups studied, along with more aggressive forms of breast cancer, such as estrogen receptor negative tumors $[5,22]$. In addition to being diagnosed with more aggressive forms of breast cancer, African-American women younger than age 50 experience higher rates of breast cancer overall compared to White women of the same age $[4,23]$. African-American women are also more likely to be diagnosed at advanced stages of the disease. However, even when diagnosed at the same disease stage, African American women face higher rates of breast cancer mortality compared to White women [23]. Estrogen receptor negative breast cancers have increased in young White women, older Hispanic women between the ages of 60 and 69, and all groups of African Americans except the eldest group $[2,23]$. Obesity and hormone use have also been linked to increased breast cancer rates [14]. There are several structural, organizational, and political factors that also affect the health care of breast cancer patients and breast cancer mortality rates [22].
Poverty, limited access to care, inadequate transportation, little to no income, and lack of health insurance increase the chances of a poor breast cancer prognosis and lower mammography screening rates [23].

Mammography among immigrant groups also varies compared to the other populations. Those who migrated to the U.S. within the last 10 years have a 33\% lower rate of mammography [22]. Unfortunately, many immigrants lack health insurance, have lower education levels, and limited income, which have been shown to contribute to lower screening rates. $[13,22]$.

In this study we examine disparities among breast cancer patients. To perform this analysis, we used cancer registries from four states: Hawaii, Washington, Utah, and Michigan. Between 1999 and 2011, Utah and Michigan had between 118.4 to 124.2 breast cancer cases per 100,000 people; and Hawaii and Washington ranged from 126.9 to 141.4 cases per 100,000 . Death rates in each state ranged from 15.5 to 22.6 per 100,000 . Hawaii and Washington had death rates of 15.5 to 19.1 per 100,000 , Utah had rates from 19.2 to 21.1 per 100,000 , and Michigan with the highest death rates of 21.2 to 22.6 per 100,000 [21].

In this study, we provide the observed numbers of breast cancer cases and deaths from 1973-2011 in the United States. We stratified by each of the four states, and provided a broad summary of breast cancer incidence, survival rates and trends, and mortality using representative sample data.

The goals of this study are to (i) determine descriptive statistics of demographic and socioeconomic variables related to the breast cancer patients' data, and (ii) obtain the hazard ratios and confidence intervals for the survival times of breast cancer patients.

\section{Materials and Methods}

Data was pulled from the Surveillance Epidemiology and End Results (SEER) database (1973-2011). The SEER database began collecting data in 1973 from approximately $10 \%$ of the U.S. population in seven states. Currently, SEER collects and publishes cancer incidence and survival information from cancer registries for $28 \%$ of the U.S. population, and includes data from twenty population-based registries [17]. This study includes information from the years 1973 to 2011 from four registries, including: Hawaii, Utah, Michigan, and Washington. A random sample of four states is considered for a small scale comparative analysis. Data from these four states were summarized for information on stage of cancer, overall survival, and the lifetime probability of developing breast cancer.

Criteria for potential eligibility in this study included: women aged 20 years and older who were registered in the SEER during 1973 to2011, had invasive stages I-III breast cancer documented in SEER as their first primary cancer diagnosis, and had no previous cancer registered in the SEER database. A participant's contribution to the person-years at risk began from the date of breast cancer diagnosis to the date of death, or loss to followup, whichever was earliest. Women who were diagnosed with 
breast cancer by autopsy were excluded. This data included 712,319 female breast cancer patients (males were excluded). The primary exposures of interest were the disparities in the characteristics of breast cancer patients and their survival data in four breast cancer registries. We used SEER-coded categories of registry ID (REG) to classify study participants into five mutually exclusive categories. A simple random sampling method was used to select 2,000 cases from each registry. For more information of selection of patients by random sampling, readers are referred to previously published literature by Khan et al [10-12]. In addition, we used subject demographic information (age at diagnosis, marital status, race and ethnicity) and survival time from the SEER dataset for statistical analysis.

A total of 8,000 females with breast cancer were included in the analysis (for each state's registry $n=2,000$ ). Survival analysis accounted for both censored (patients who survived until the end of SEER registry's cutoff date) and uncensored (any patient who died within the SEER registry's cutoff date) data. Survival time was calculated in months using the Cox Proportional Hazards model, adjusting for age at diagnosis, race, ethnicity, and marital status for included registries. Cox Proportional Hazards models generated the adjusted Hazard Ratio (HR) and their 95\% confidence intervals (CI). Data analysis was conducted by using SPSS software (IBM SPSS for Windows version 20, 2011) and SAS ${ }^{\circledR}$ software version 9.4.

\section{Results and Discussion}

Using a simple random sampling method, 2,000 patients were selected from each of the four state cancer registries (Hawaii, Michigan, Utah, and Washington). Tables 1 and 2 contain the descriptive statistics from this analytical sample.

Table 1 shows: ages at diagnosis (in years), survival time (in months), and at the time of diagnosis. It also contains the descriptive statistics for this analytical sample. Data shows Washington exhibits the highest mean age of diagnosis, 61.46

Table 1: Age at Diagnosis, and Survival Time for Female Breast Cancer Patients $(n=2,000)$

\begin{tabular}{|l|l|l|l|l|l|}
\hline & Statistics & Hawaii & Michigan & Utah & Washington \\
\hline $\begin{array}{l}\text { Age at } \\
\text { diagnosis } \\
\text { (years) }\end{array}$ & Mean & 58.64 & 60.98 & 60.77 & 61.46 \\
\hline & SE & 0.310 & 0.317 & 0.323 & 0.321 \\
\hline & Median & 57 & 61 & 60.5 & 61 \\
\hline & Quartiles & $\begin{array}{l}48,56, \\
71\end{array}$ & $50,61,72$ & $\begin{array}{l}50,60.50, \\
72\end{array}$ & $50,61,72$ \\
\hline $\begin{array}{l}\text { Survival } \\
\text { time } \\
\text { (months) }\end{array}$ & Mean & 118.21 & 152.67 & 131.94 & 151.6 \\
\hline & SE & 4.861 & 14.955 & 11.241 & 14.110 \\
\hline & Median & 90 & 85 & 82.5 & 90.5 \\
\hline & Quartiles & $\begin{array}{l}36,89, \\
165\end{array}$ & $\begin{array}{l}32,85, \\
160.75\end{array}$ & $\begin{array}{l}34,82.50, \\
156\end{array}$ & $\begin{array}{l}37,90.50, \\
168.75\end{array}$ \\
\hline
\end{tabular}

years (Standard Error $(\mathrm{SE})=0.321$ ), followed by Michigan, Utah, and Hawaii, with mean age of diagnosis being, 60.98 (SE =0.317), 60.77 ( $\mathrm{SE}=0.323$ ), and 58.64 ( $\mathrm{SE}=0.305$ ), respectively. The 25 th and 50th quartiles of age at diagnosis generally ranged from 4850 to 56-61 years of age, respectively across for all four states. The mean survival days (in months) were also calculated and stratified by each cancer registry. Michigan reported the longest mean survival time of 152.67 months (SE $=14.955$ ), and Hawaii reported the shortest mean survival time of 118.21 (SE=4.861) months, representing a 34.46 months difference (2.87 years). Therefore, statewide health policies may affect long-term survival, access and use of care, and prognosis of those diagnosed with breast cancer. The 25 th and 50 th quartiles of survival times generally ranged from 32-37 to 83-91 months, respectively, for all four states.

Table 2 shows the frequency and percentage of each race, ethnicity, and marital status for each of the four states studied. The majority of participants were married, followed by widowed being the second most common relationship status. Whites were reported as the predominant racial category among three out of the four states, with the exception of Hawaii, which reported a larger Asian or Pacific Islander population. Blacks were reported as the second most common race for Michigan (20.45\%) of women with a breast cancer diagnosis. Asian and Pacific Islanders had the second most reported cases in Utah (1.75\%), and Washington (4.35\%). American Indians/Alaskan Natives were the first race diagnosed for breast cancer in Washington (1.3\%).

Table 3 shows the analysis of maximum likelihood estimates. From Table 3, two of the four states reported statistical significance in survival rates. Hawaii, with the highest mean survival time, was made the referent group for calculating hazard ratios, adjusting for states using the Cox Proportional Regression. Hazard ratios compare the probability of an event occurring in one group versus another group taking into account the time elapsed until the event occurs. Hazard ratios were calculated for three states, where the hazard ratio is the estimate of the ratio of the hazard rate in one group to the hazard rate in the referent group, Hawaii. Statistical significance was established if the $95 \%$ confidence interval did not include the integer 1 . The hazard ratio for Michigan is 1.532 with a $95 \%$ confidence interval $(1.391,1.686)$ and its $p$-value $=0.0001$. The hazard ratio for Utah is 1.346 with a $95 \%$ confidence interval $(1.219,1.486)$ and its $p$ $=0.0001$. The hazard ratio for Washington is 1.231 with a $95 \%$ confidence interval $(1.114,1.360)$ and its $p=0.0001$.

The mean age at diagnosis ranged from age 58.86 to 61.46 , underlining the importance for women to follow their doctor's recommendations for mammography screening. Breast cancer takes approximately 2 to 5 years to develop; therefore, women should be screened regularly to identify and treat pre-cancers and to prevent the progression of breast cancer. The findings in this study give credence to the importance of early detection and treatment in reducing breast cancer. Increased efforts should be made to lengthen the survival time for breast cancer patients, particularly for those in racial and ethnic groups that face shorter survival times. 
Table 2: Race, Ethnicity and Marital Status for Female Breast Cancer Patients $(n=2,000)$

\begin{tabular}{|c|c|c|c|c|c|c|c|c|c|}
\hline & & HI & $\%$ & MI & $\%$ & UT & $\%$ & WA & $\%$ \\
\hline \multirow[t]{6}{*}{ Race } & White & 602 & 30.2 & 1563 & 78.15 & 1945 & 97.25 & 1833 & 91.65 \\
\hline & Black & 19 & 0.85 & 409 & 20.45 & 9 & 0.45 & 52 & 2.6 \\
\hline & American Indian or Alaskan Native & 3 & 0.2 & 0 & 0 & 5 & 0.25 & 26 & 1.3 \\
\hline & Asian or Pacific Islander & 1367 & 68.3 & 18 & 0.9 & 35 & 1.75 & 87 & 4.35 \\
\hline & Other & 3 & 0.2 & 5 & 0.25 & 0 & 0 & 1 & 0.05 \\
\hline & Unknown & 6 & 0.25 & 5 & 0.25 & 6 & 0.3 & 1 & 0.05 \\
\hline \multirow[t]{2}{*}{ Ethnicity } & Hispanic & 79 & 3.9 & 25 & 1.25 & 66 & 3.3 & 27 & 1.35 \\
\hline & Non-Hispanic & 1921 & 96.1 & 1975 & 98.75 & 1934 & 96.7 & 1973 & 98.65 \\
\hline \multirow[t]{6}{*}{ Marital Status } & Single & 214 & 10.95 & 223 & 11.15 & 119 & 5.95 & 171 & 8.55 \\
\hline & Married & 1197 & 59.6 & 1013 & 50.65 & 1288 & 64.4 & 1159 & 57.95 \\
\hline & Separated & 14 & 0.75 & 14 & 0.7 & 6 & 0.3 & 9 & 0.45 \\
\hline & Divorced & 187 & 9.3 & 203 & 10.15 & 165 & 8.25 & 210 & 10.5 \\
\hline & Widowed & 323 & 16.4 & 454 & 22.7 & 378 & 18.9 & 361 & 18.05 \\
\hline & Unknown & 65 & 3 & 93 & 4.65 & 44 & 2.2 & 90 & 4.5 \\
\hline
\end{tabular}

Table 3: Hazard Ratio and Confidence Intervals

\begin{tabular}{|c|c|c|c|c|c|c|c|c|}
\hline States & DF & $\begin{array}{c}\text { Parameter } \\
\text { Estimate }\end{array}$ & $\begin{array}{c}\text { Standard } \\
\text { Error (SE) }\end{array}$ & Chi-Square & Pr $>$ ChiSq & $\begin{array}{c}\text { Hazard } \\
\text { Ratio }\end{array}$ & $\begin{array}{c}\text { 95\% Hazard Ratio } \\
\text { Confidence } \\
\text { Limits }\end{array}$ \\
\hline Michigan & 1 & 0.42628 & 0.04896 & 75.8080 & $p<.0001$ & 1.532 & $(1.391$ & $1.686)$ \\
\hline Utah & 1 & 0.29722 & 0.05041 & 34.7713 & $p<.0001$ & 1.346 & $(1.219$ & $1.486)$ \\
\hline Washington & 1 & 0.20780 & 0.05097 & 16.6202 & $p<.0001$ & 1.231 & $(1.114$ & $1.360)$ \\
\hline
\end{tabular}

In Table 3, we used Hawaii as the referent group. Statistical significance is established if the 95\% confidence interval did not include 1. Hawaii had a significantly increased risk of death compared to Michigan (Hazard ratio: 1.532; 95\% Confidence Limits: 1.391-1.686); Utah (Hazard ratio: 1.346; 95\% Confidence Limits: 1.219-1.486); and Washington (Hazard ratio: 1.231; 95\% Confidence Limits: 1.114-1.360).

Although this study has its strengths, such as the use of a nationally recognized cancer database, SEER, there are limitations. SEER has collected and published critical cancer statistics for over thirty years from cancer registries throughout the United States, and is considered a reliable source of information on incidence, mortality, and other related variables. However, it lacks insight into the other variables such as social and economic factors that affect the survival time of these states.

\section{Conclusions}

There are several factors accounting for breast cancer survival, including state, local, and individual level factors which support differences in breast cancer survival based on geographic region. For example, the breast cancer survival was significantly different for those living in Michigan compared to Hawaii with an almost three year difference in disease morbidity. It is imperative to understand the differences found in breast cancer prevention between the two states in order to identify successful prevention tactics. Additionally, it is necessary to understand other factors which may account for differences in patient survival.

Future studies should compare the statewide policies, preventive breast cancer systems, and the current state of the health care systems to identify their impact on breast cancer stage at diagnosis and prognosis. However, it is important for future studies to address other demographic variables including income, education level, and health insurance status. In order to better understand patient survival, primary factors affecting patient prognosis should be addressed. These factors can be current medical treatment and prevention regimen that impact breast cancer diagnosis and treatment, as well as more indirect factors that will also affect survival. Further investigation should also be considered at the community level to determine what specific factors may prevent receipt of preventive screening or access to adequate care. This will inform the design and troubleshooting of prevention and treatment efforts primarily in reaching the most "at-risk" groups. These findings give credence to the importance of early detection and treatment and targeting underserved populations in order to reduce breast cancer incidence nationwide. Given the patient's current location and 
reported conditions, the findings may encourage healthcare providers and researchers to gather information about geographical and local characteristics that affect the survival rates of breast cancer patients.

The mean range of age at diagnosis was 58.64 to 61.46 , which aligns well with the recommended breast cancer screening. However, it is important to recognize that some groups experience earlier presentations of breast cancer and should be screened earlier than the standard guidelines. Health care and public health efforts should target the most disadvantaged communities to help improve breast cancer related health equity. For example, African American women experience more aggressive forms of breast cancer at early ages; therefore, this group may require earlier screening and education efforts. However, all women need to be screened consistently. Furthermore, economically disadvantaged patients and those lacking health insurance often do not receive adequate or appropriate care for their diagnosis, adversely affecting their prognosis. This may include improving the treatment options for these groups and improving screening efforts to ensure earlier disease detection. Further research is needed to identify genetic, environmental, and socioeconomic risk factors that contribute to breast cancer.

\section{Acknowledgements}

The authors are grateful to the National Cancer Institute in the United States for allowing them access to the SEER's database. There is no financial support for this study.

\section{References}

1. Althius MD, Dozier JM, Anderson WF, Devesa SS, Brinton LA. Global trends in breast cancer incidence and mortality 1973-1997. Int J Epidemiol. 2005;34(2):405-412.

2. Clarke CA, Keegan THM, Yang J, Press DJ, Kurian AW, Patel AH, et al. Age-specific incidence of breast cancer subtypes: understanding the black-white crossover. JNCI. 2012;104(14):1094-1101. doi: 10.1093/ jnci/djs264

3. Coughlin SS, Ekueme DU. Breast cancer as a global health concern. Cancer Epidemiol. 2009;33(5):315-318. doi: 10.1016/j. canep.2009.10.003

4. DeSantis C, Ma J, Bryan L, Ahmedin Jemal. Breast cancer statistics, 2013. CA Cancer J Clin. 2014;64(1):52-62.

5. Dunn BK, Agurs-Collins T, Browne D, Lubet R, Johnson KA. Health disparities in breast cancer: biology meets socioeconomic status. Breast Cancer Res Treat. 2010;121(2):281-292. doi: 10.1007/s10549010-0827-x

6. Gross CP, Long JB, Ross JS, Abu-Khalaf MM, Wang R, Killelea $\mathrm{BK}$,et al. The cost of breast cancer screening in the Medicare population. JAMA Intern Med. 2013;173(3):220-226. doi: 10.1001/ jamainternmed.2013.1397.

7. Howard DH, Tangka FKL, Royalty J, Dalzell LP, Miller J, O'Hara B, et al. Breast cancer screening of underserved women in the USA results from the National Breast and Cervical Cancer Early Detection Program, 1998-2012. Cancer Causes Control. 2015;26:657-668. doi: 10.1007/s10552-015-0553-0.

8. Jemal A, Bray F, Center MM, Ferlay J, Ward E, Forman D. Global cancer statistics. CA Cancer J Clin. 2011;61(2):69-90. doi: 10.3322/ caac.20107.

9. Khan HMR, Saxena A, Gabbidon K, Rana S, Ahmed NU. Model-based survival estimates of female breast cancer data. Asia Pac J Public Health. 2014;15(6):2893-2900.

10. Khan HMR, Saxena A, Gabbidon K, Elizabeth Ross, Alice Shrestha. Statistical applications for the prediction of white Hispaic breast cancer survival. Asia Pav J Cancer Prevention. 2014;15(14):55715575. DOI: 10.7314/APJCP.2014.15.14.5571

11. Khan HMR, Saxena A, Gabbidon K, Stewart TS, Bhatt C. Survival analysis for white non-Hispanic female breast cancer patients. Asia Pac J Cancer Prevention. 2014;15(9):4049-4054.

12. Khan HMR, Saxena A, Vera V, Gabbidon K, Perea N, Stewart TS, et al. Black Hispanic and Black Non-Hispanic Breast Cancer Survival Data Analysis with Half-normal Model Application. Asia Pac J Cancer Prevention. 2014;15(21):9453-9458.

13. Miranda PY, Tarraf W, Gonzalez HM, Michelle Johnson-Jennings, Hector M González. Breast cancer screening and ethnicity in the United states: implications for health disparities research. Breast Cancer Res Treat. 2011;128(2):535-542. doi: 10.1158/1055-9965.EPI-11-0873

14. Naraynsingh V, Harihan S, Dan D, Bhola S, Nagee K. Trends in breast cancer mortality in Trinidad and Tobago--a 35-year study. Cancer Epidemiol. 2010;34(1):20-23. doi: 10.1016/j.canep.2009.11.003.

15. Pruitt SL, Lee SJC, Tiro JA, Xuan L, Ruiz JM, Inrig S. Residential racial segregation and mortality among black, white and Hispanic urban breast cancer patients in Texas, 1995 to 2009. Cancer. 2015;121(11):1845-1855. doi: 10.1002/cncr.29282.

16. Surveillance, Epidemiology, and End Results (SEER) Program. Research Data (1973-2011), (2013, accessed 30 September 2015).

17. Siegel R, DeSantis C, Virgo K, Stein K, Mariotto A, Smith T, et al. Cancer Treatment and Survivorship Statistics, 2012. CA Cancer J Clin. 2012;62(4):220-241. doi: 10.3322/caac.21149

18. Siegel R, Ma J, Zou Z. Cancer Statistics, 2014. CA Cancer J Clin. 2014;64(1):9-29. doi: 10.3322/caac.21208

19. Surveillance, Epidemiology and End Results. SEER stat fact sheet cervix uteri. (2013, accessed 30 September 2015).

20.Tian N, Wilson JG, Zhan FB. Spatial association of racial/ethnic disparities between late-stage diagnosis and mortality for female breast cancer: where to intervene? Int J Health Geographics. 2011;10(1):24. DOI: 10.1186/1476-072X-10-24

21.Center for Disease Control and Prevention. United States Cancer Statistics 1999-2012 Incidence and Mortality Web-based report. Available at: http://www.cdc.gov/uscs. (2015, accessed 30 September 2015).

22. Ward E, Jemal A, Cokkinides V, Singh GK, Cardinez C, Ghafoor A, et al. Cancer Disparities by Race/ Ethnicity and Socioeconomic Status. Cancer Disparities. 2004; 54(2):78-93

23. Wheeler SB, Reeder-Hayes KE, Carey L. Disparities in breast cancer treatment and outcomes: biological, social, and health system determinants and opportunities for research. Oncologist. 2013;18(9):986-993. doi: 10.1634/theoncologist.2013-0243

24. Youlden DR, Cramb SM, Dunn N, Muller JM, Pyke CM, Baade PD. The descriptive epidemiology of female breast cancer: an international comparison of screening, incidence, survival, and mortality. Cancer Epidemiol. 2012;36(3): 237-248. doi: 10.1016/j.canep.2012.02.007. 